\title{
Nonstandard micro-inertia terms in the relaxed micromorphic model: well-posedness for dynamics
}

\author{
Sebastian Owczarek ${ }^{1}$ and Ionel-Dumitrel Ghiba ${ }^{2}$ and Marco-Valerio d'Agostino ${ }^{3}$ and \\ Patrizio Neff ${ }^{4}$
}

February 12, 2019

\begin{abstract}
We study the existence of solutions arising from the modelling of elastic materials using generalized theories of continua. In view of some evidence from physics of meta-materials we focus our effort on two recent nonstandard relaxed micromorphic models including novel micro-inertia terms. These novel microinertia terms are needed to better capture the band-gap response. The existence proof is based on the Banach fixed point theorem.
\end{abstract}

Mathematics Subject Classification: 35M33, 35Q74, 74H20, 74M25, 74B99

Keywords: wave propagation, generalised continua, micro-inertia

\section{Contents}

1 Introduction 1

1.1 Preliminaries and motivation .......................... 1

2 Relaxed micromorphic models including micro-inertia terms 4

2.1 Description of the mechanical model . . . . . . . . . . . . . . . . . . . . . . . . 4

2.2 Existence of the solution . . . . . . . . . . . . . . . . . . . . . . . . . 7

3 On the existence for a simplified model 11

4 Final remarks

5 Acknowledgement $\quad 13$

\section{Introduction}

\subsection{Preliminaries and motivation}

The micromorphic theory [19, 39] is a generalised theory of continua which, in order to describe both macro- and micro-deformation, considers that any point of the body is endowed with two fields, a vector field $u: \Omega \times[0, T] \rightarrow$ $\mathbb{R}^{3}$ for the displacement of the macroscopic material points, and a tensor field $P: \Omega \times[0, T] \rightarrow \mathbb{R}^{3 \times 3}$ describing

\footnotetext{
${ }^{1}$ Sebastian Owczarek, Faculty of Mathematics and Information Science, Warsaw University of Technology, ul. Koszykowa 75, 00-662 Warsaw, Poland; email: s.owczarek@mini.pw.edu.pl

${ }^{2}$ Ionel-Dumitrel Ghiba, Alexandru Ioan Cuza University of Iaşi, Department of Mathematics, Blvd. Carol I, no. 11, 700506 Iaşi, Romania; Octav Mayer Institute of Mathematics of the Romanian Academy, Iaşi Branch, 700505 Iaşi; email: dumitrel.ghiba@uaic.ro

${ }^{3}$ Marco-Valerio d'Agostino, Laboratoire de Génie Civil et Ingénierie Environnementale, Université de Lyon-INSA, Bâtiment Coulomb, 69621 Villeurbanne Cedex, France, email: marco-valerio.dagostino@insa-lyon.fr

${ }^{4}$ Patrizio Neff, Head of Lehrstuhl für Nichtlineare Analysis und Modellierung, Fakultät für Mathematik, Universität DuisburgEssen, Campus Essen, Thea-Leymann Str. 9, 45127 Essen, Germany, email: patrizio.neff@uni-due.de
} 
the micro-deformation (micro-distortion) of the substructure of the material. This theory was introduced 58 years ago by Eringen and Mindlin and an important part of its reason to be was to have a model which is capable to improve agreement between the analytical and numerical results regarding wave propagation indicated by the model and those obtained in experiments on actual materials. When the classical theory of linear elasticity is used, no dispersive effects can be predicted. However, the elastic wave propagation through heterogeneous media is generally dispersive: each wave number travels with a distinct wave speed. Some other possible applications of the micromorphic model are described in [17].

It is well-known that the Cosserat theory (the case when the micro-distortion $P$ is antisymmetric) is a particular case of the micromorphic theory. The Cosserat theory was introduced in 1909 [12] (see also [43, 31, 48, 41, 42]) but it was not really taken into account until the micromorphic theory was introduced. The biggest shortcoming of the classical micromorphic theory is that it involves an excessively large number of constitutive coefficients, which must be determined if one wants to use it in applications. In order to avoid this shortcoming, one solution is to adapt the micromorphic theory to the phenomena we want to model. This is done for instance in the works [44, 22] in which $P$ is assumed to be symmetric (the so called microstrain model).

In a recent paper [46], we have introduced a modified micromorphic theory which we called the relaxed micromophic model. This model completes the model which was proposed in [11 and which was critically discussed in [59. Since Claus and Eringen have introduced such a model having in mind its application to the dislocation theory, the main criticism centered about the non-symmetry of the force stress tensor. At that time it was not clear that such a theory may have a chance to be well-posed, even when the force stress tensor is symmetric. However, due to some new Poincaré-Korn type inequalities [6, 51], the relaxed micromorphic model is well-posed also when the force stress tensor is symmetric [24]. We have explained in [45] why the relaxed model is a particular case of the classical Mindlin-Eringen model and how the energy of the relaxed model may be obtained taking some suitable form of the constitutive coefficients in the classical theory. The relaxed micromorphic theory is still general enough to incorporate the kinematics of the most used particular theories of the classical micromorphic theory, see [46] and Figure 1].

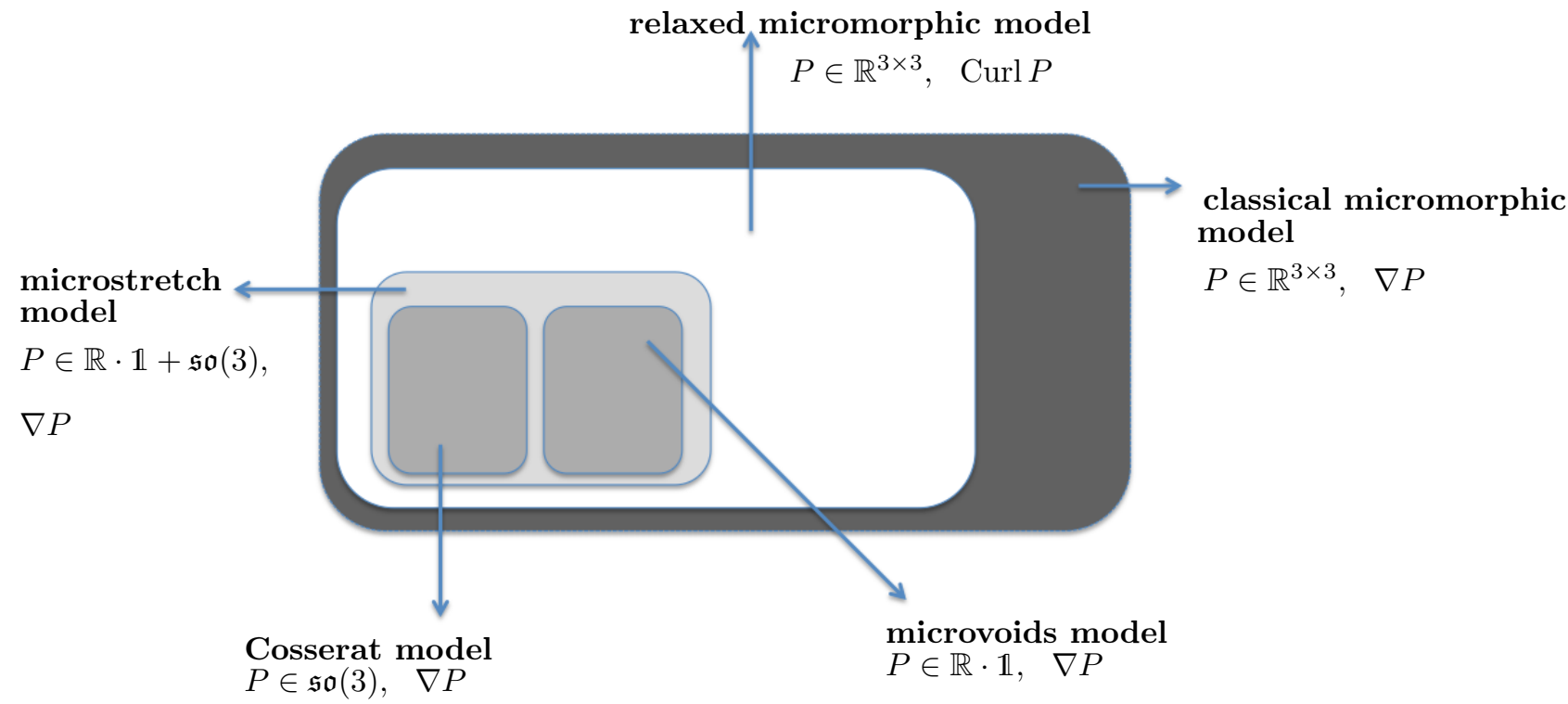

Figure 1: An illustration of the relations between different theories of materials with microstructure: the classical micromorphic model [19]; the linear microstretch model [17]; the linear isotropic Cosserat model [12]; the linear isotropic microvoids model [52]. Comparison with other models are discussed in [46].

Considering a wave ansatz in the partial differential equations arising in the relaxed micromorphic model, some band gaps arise in the diagrams describing the dispersion curves. This means that there exists an interval of wave frequencies for which no wave propagation may occur [37, 36, 38. Such an interesting phenomena is obtained when meta-materials are designed and may not be captured by the classical micromorphic approach [14, 35. These meta-materials are able to "absorb" or even "bend" elastic waves with no energetic cost (see e.g. [20, 21]). They are conceived arranging small components into periodic or quasi-periodic patterns in such a way 
that the resulting structure possesses new unimaginable properties with respect to the original material. This insight has opened a way to obtain new generalized continuum models allowing to describe the behavior of metastructures in the simplified framework of continuum mechanics with homogenized material behavior. Moreover, due to the a priori relation between macro and micro parameters of the considered samples obtained in [4], the constitutive parameters of our model can be identified on real meta-materials [5, 13] even for anisotropic materials, opening the way to the efficient design and realization of meta-structures.

Besides the interesting property of the relaxed micromorphic theory to model certain band-gap metamaterials, the mathematical results which can be obtained are also surprising: it is noted that all the results which assume that the energy is positive definite in the relaxed model are in fact valid without assuming that the corresponding energy is positive definite in the classical Mindlin-Eringen model. Therefore, for the relaxed model we have obtained [24] extensions of the results established by Sóos [58, by Hlaváček [27, by Ieşan and Nappa [29] and by Ieşan [30] which all use the positive definitness in the Mindlin-Eringen model. We mention also the results given by Picard et al. [56] which have proved an existence result in the linear theory of micromorphic elastic solids using an interesting mother/descendant mechanism regarding various models. On the other hand, almost all the mathematical results are obtained in the classical Mindlin-Eringen model assuming a strong anchoring boundary condition: the microdistortion $P$ is completely described at one part of the boundary. Our model gives us the possibility to relax this condition and to be able to prescribe only the tangential components of the rows of $P$.

Going back to the applications of the relaxed micromorphic model in the modelling of wave propagation in meta-materials, it can be observed that for large wavenumbers the dispersion curves obtained from our model have a different behavior than those which are predicted by the Bloch-Floquet analysis. By introducing some nonstandard micro-inertia terms in the form of the kinetic energy the fitting between the dispersion curves given by the Bloch-Floquet analysis and by the new model is considerably improved [34, 33, 13, 1, In a recent paper [5] it is shown that while the linear elastic Cauchy continuum is able to describe the pulse propagation through the micro-structured domain only in the low frequency regime, the relaxed micromorphic model with these nonstandard micro-inertia terms is able to account for the overall behavior of the material for frequency regimes including the band-gap and higher frequencies. Moreover, this property seems to be difficult to obtain with other generalized continuum models.

The idea of considering some new terms in the form of the kinetic energy is not entirely new, it already appeared in the linear theory of nonlocal elasticity introduced in [16] and derived from a more general theory developed by Eringen and Edelen [18. Gradient elasticity can also be used to capture the dispersive behavior of waves that propagate through a heterogeneous material and the presence of micro-inertia terms was used, before the work of Eringen [16], also by Mindlin [39, page 68]. The difference is that Mindlin used micro-inertia terms only in the context of gradients elasticity, while Eringen used them without considering higher order derivatives of the displacement [3]. Various formats of gradient elasticity with inertia gradients have been considered in many works [57, 60, 10, 15, 7, 26]. But there does not seem to exist a rigorous mathematical treatment of these models.

The main aim of this paper is to present relaxed micromorphic models including some nonstandard microinertia terms and to prove that these models are well-posed. Our analysis covers also the case when the stress tensor is symmetric since, in our mathematical approach, the presence of the constitutive term which makes it non-symmetric is not essential: for the mathematical analysis of the model the presence of the Cosserat couple modulus is redundant. However, in the study of meta-materials this coefficient is essential when one wants to model the band-gap phenomena [37.

The plan of the paper is now the following. In Section 2 we introduce the initial-boundary value problem arising in the model when non-standard inertia terms are present and we prove the existence of the solution. The used approach is not common in elastodynamics, it is based on the Banach-fixed point theorem and it was suggested by an approach from thermo-visco-plasticity [9]. We mention that the usual approaches based on the semigroup theory of linear operators or on the Galerkin method seem to not be able to lead to an existence result without assuming a priori some compatibilities between the domains of the operators involved in our partial differential equations. We avoid these assumptions, which, from a mechanical point of view lead to a very particular model which is not capable to describe the behavior of waves in meta-materials, i.e. it does not correspond to the main raison d'être of our model. In the last section, we introduce a simplified model and we prove that the corresponding initial-boundary value problem is still well-posed. 


\section{Relaxed micromorphic models including micro-inertia terms}

We introduce a new model which takes into account the influence of some nonstandard micro-inertia terms on the behavior of the solution of the obtained initial-boundary value problem.

We consider a micromorphic continuum which occupies a bounded domain $\Omega$ having a piecewise smooth surface $\partial \Omega$. The motion of the body is referred to a fixed system of rectangular Cartesian axes $O x_{i},(i=1,2,3)$. Throughout this paper (if we do not specify otherwise) Latin subscripts take the values $1,2,3$.

We denote by $\mathbb{R}^{3 \times 3}$ the set of real $3 \times 3$ matrices. For all $X \in \mathbb{R}^{3 \times 3}$ we set $\operatorname{sym} X=\frac{1}{2}\left(X^{T}+X\right)$ and skew $X=\frac{1}{2}\left(X-X^{T}\right)$. For $a, b \in \mathbb{R}^{3}$ we let $\langle a, b\rangle_{\mathbb{R}^{3}}$ denote the scalar product on $\mathbb{R}^{3}$ with associated vector norm $\|a\|_{\mathbb{R}^{3}}^{2}=\langle a, a\rangle_{\mathbb{R}^{3}}$. The standard Euclidean scalar product on $\mathbb{R}^{3 \times 3}$ is given by $\langle X, Y\rangle_{\mathbb{R}^{3 \times 3}}=\operatorname{tr}\left(X Y^{T}\right)$, and thus the Frobenius tensor norm is $\|X\|^{2}=\langle X, X\rangle_{\mathbb{R}^{3 \times 3}}$. In the following we omit the index $\mathbb{R}^{3}, \mathbb{R}^{3 \times 3}$. The identity tensor on $\mathbb{R}^{3 \times 3}$ will be denoted by $\mathbb{1}$, so that $\operatorname{tr}(X)=\langle X, \mathbb{1}\rangle$. We adopt the usual abbreviations of Lie-algebra theory, i.e., $\mathfrak{s o}(3):=\left\{X \in \mathbb{R}^{3 \times 3} \mid X^{T}=-X\right\}$ is the Lie-algebra of skew symmetric tensors and $\operatorname{Sym}(3)$ denotes the set of symmetric tensors.

By $C_{0}^{\infty}(\Omega)$ we denote the set of smooth functions with compact support in $\Omega$. All the usual Lebesgue spaces of square integrable functions, vector or tensor fields on $\Omega$ with values in $\mathbb{R}, \mathbb{R}^{3}$ or $\mathbb{R}^{3 \times 3}$, respectively will be generically denoted by $L^{2}(\Omega)$. Moreover, we use the standard Sobolev spaces [2, 32, 25]

$$
\begin{array}{ll}
\mathrm{H}^{1}(\Omega)=\left\{u \in L^{2}(\Omega) \mid \operatorname{grad} u \in L^{2}(\Omega)\right\}, & \|u\|_{\mathrm{H}^{1}(\Omega)}^{2}:=\|u\|_{L^{2}(\Omega)}^{2}+\|\operatorname{grad} u\|_{L^{2}(\Omega)}^{2}, \\
\mathrm{H}(\operatorname{curl} ; \Omega)=\left\{v \in L^{2}(\Omega) \mid \operatorname{curl} v \in L^{2}(\Omega)\right\}, & \|v\|_{\mathrm{H}(\operatorname{curl} ; \Omega)}^{2}:=\|v\|_{L^{2}(\Omega)}^{2}+\|\operatorname{curl} v\|_{L^{2}(\Omega)}^{2},
\end{array}
$$

of functions $u$ or vector fields $v$, respectively. Furthermore, we introduce their closed subspaces $\mathrm{H}_{0}^{1}(\Omega)$, and $\mathrm{H}_{0}(\operatorname{curl} ; \Omega)$ as the closure with respect to the associated graph norms of $C_{0}^{\infty}(\Omega)$. Roughly speaking, $\mathrm{H}_{0}^{1}(\Omega)$ is the subspace of functions $u \in \mathrm{H}^{1}(\Omega)$ which are zero on $\partial \Omega$, while $\mathrm{H}_{0}(\operatorname{curl} ; \Omega)$ is the subspace of vectors $v \in \mathrm{H}(\operatorname{curl} ; \Omega)$ which are normal at $\partial \Omega$ (see [51, 49, 50]). For vector fields $v$ with components in $\mathrm{H}^{1}(\Omega)$ and tensor fields $P$ with rows in $\mathrm{H}(\operatorname{curl} ; \Omega)$, i.e.,

$$
v=\left(v_{1}, v_{2}, v_{3}\right)^{T}, \quad v_{i} \in \mathrm{H}^{1}(\Omega), \quad P=\left(P_{1}^{T}, P_{2}^{T}, P_{3}^{T}\right)^{T}, \quad P_{i} \in \mathrm{H}(\operatorname{curl} ; \Omega),
$$

we define

$$
\nabla v:=\left(\left(\operatorname{grad} v_{1}\right)^{T},\left(\operatorname{grad} v_{2}\right)^{T},\left(\operatorname{grad} v_{3}\right)^{T}\right)^{T}, \quad \operatorname{Curl} P:=\left(\left(\operatorname{curl} P_{1}\right)^{T},\left(\operatorname{curl} P_{2}\right)^{T},\left(\operatorname{curl} P_{3}\right)^{T}\right)^{T} .
$$

We note that $v$ is a vector field, whereas $P, \operatorname{Curl} P$ and $\nabla v$ are second order tensor fields. The corresponding Sobolev spaces will be denoted by $\mathrm{H}^{1}(\Omega)$ and $\mathrm{H}(\mathrm{Curl} ; \Omega)$, and $\mathrm{H}_{0}^{1}(\Omega)$ and $\mathrm{H}_{0}(\mathrm{Curl} ; \Omega)$, respectively.

For a fourth order tensor $\mathbb{C}$ and $X \in \mathbb{R}^{3 \times 3}$, we have $\mathbb{C} . X \in \mathbb{R}^{3 \times 3}$ with the components $(\mathbb{C} . X)_{i j}=\mathbb{C}_{i j k l} X_{k l}$, while for a sixth order tensor $\mathbb{L}$ we consider $\mathbb{L} . Z \in \mathbb{R}^{3 \times 3 \times 3}$ for all $Z \in \mathbb{R}^{3 \times 3 \times 3},(\mathbb{L} . Z)_{i j k}=\mathbb{L}_{i j k m n p} Z_{m n p}$, where Einstein's summation rule is used.

\subsection{Description of the mechanical model}

The mechanical model is formulated in the variational context. This means that we consider an action functional on an appropriate function-space. The space of configurations of the problem is

$$
\mathcal{Q}:=\left\{(u, P) \in C^{1}\left(\bar{\Omega} \times[0, T], \mathbb{R}^{3}\right) \times C^{1}\left(\bar{\Omega} \times[0, T], \mathbb{R}^{3 \times 3}\right):(u, P) \text { verifies conditions }\left(\mathrm{B}_{1}\right) \text { and }\left(\mathrm{B}_{2}\right)\right\}
$$

where

- $\left(\mathrm{B}_{1}\right)$ are the boundary conditions $u(x, t)=\varphi(x, t)$ and $P_{i}(x, t) \times n=\psi_{i}(x, t), i=1,2,3, \quad(x, t) \in$ $\partial \Omega \times[0, T]$, where $n$ is the unit outward normal vector on $\partial \Omega \times[0, T], P_{i}, i=1,2,3$ are the rows of $P$ and $\varphi, \psi_{i}$ are prescribed continuous functions and $[0, T]$ is the time interval;

- $\left(\mathrm{B}_{2}\right)$ are the initial conditions $\left.u\right|_{t=0}=u_{0},\left.u_{, t}\right|_{t=0}=\underline{u}_{0},\left.P\right|_{t=0}=P_{0},\left.P_{, t}\right|_{t=0}=\underline{P}_{0}$ in $\Omega$, where $u_{0}(x), \underline{u}_{0}(x)$, $P_{0}(x), \underline{P}_{0}(x)$ are prescribed smooth functions. 
The action functional $\mathscr{A}: \mathcal{Q} \rightarrow \mathbb{R}$, is the sum of the internal and external action functionals $\mathscr{A}_{\mathscr{L}}^{\text {int }}, \mathscr{A}^{\text {ext }}: \mathcal{Q} \rightarrow \mathbb{R}$ defined as follows

$$
\begin{aligned}
& \mathscr{A}_{\mathscr{L}}^{\text {int }}[(u, P)]:=\int_{0}^{T} \int_{\Omega} \mathscr{L}\left(u, t, P_{, t}, \nabla u, t, \operatorname{Curl} P_{, t}, \nabla u, P, \operatorname{Curl} P\right) d v d t, \\
& \mathscr{A}^{\text {ext }}[(u, P)]:=\int_{0}^{T} \int_{\Omega}(\langle f, u\rangle+\langle M, P\rangle) d v d t,
\end{aligned}
$$

where $\mathscr{L}$ is the Lagrangian density of the system and $f, M$ are the body force and double body force, and comma followed by a subscript denotes the time derivative. We recommend also the paper by Germain 23. for some explanations about the physical significance of the involved quantities. In order to find the stationary points of the action functional, we have to calculate its first variation:

$$
\delta \mathscr{A}=\delta \mathscr{A}_{\mathscr{L}}^{i n t}=\delta \int_{0}^{T} \int_{\Omega} \mathscr{L}\left(u_{, t}, P_{, t}, \nabla u_{, t}, \operatorname{Curl} P_{, t}, \nabla u, P, \operatorname{Curl} P\right) d v d t
$$

For the Lagrangian energy density we assume the standard split in kinetic minus potential energy density:

$$
\mathscr{L}\left(u_{, t}, P_{, t}, \nabla u_{, t}, \operatorname{Curl} P_{, t}, \nabla u, P, \operatorname{Curl} P\right)=K\left(u_{, t}, P_{, t}, \nabla u_{, t}, \operatorname{Curl} P_{, t}\right)-W(\nabla u, P, \operatorname{Curl} P),
$$

In general anisotropic linear elastic relaxed micromorphic homogeneous media, we consider that the kinetic energy and the potential energy density have the following expressions

$$
\begin{aligned}
& K\left(u_{, t}, P_{, t}, \nabla u_{, t}, \operatorname{Curl} P_{, t}\right)=\frac{1}{2}\left\langle\rho u_{, t}, u_{, t}\right\rangle+\frac{1}{2}\left\langle J P_{, t}, P_{, t}\right\rangle+\frac{1}{2}\left\langle\widetilde{\mathbb{C}}_{e} \cdot \operatorname{sym}\left(\nabla u_{, t}-P_{, t}\right), \operatorname{sym}\left(\nabla u_{, t}-P_{, t}\right)\right\rangle \\
& +\frac{1}{2}\left\langle\widetilde{\mathbb{C}}_{c} \cdot \text { skew }\left(\nabla u_{, t}-P_{, t}\right), \text { skew }\left(\nabla u_{, t}-P_{, t}\right)\right\rangle \\
& +\frac{1}{2}\left\langle\widetilde{\mathbb{C}}_{\text {micro }} \cdot \operatorname{sym} P_{, t}, \operatorname{sym} P_{, t}\right\rangle+\mu \frac{L_{c}^{2}}{2}\left\langle\widetilde{\mathbb{L}}_{\text {aniso }} \cdot \operatorname{Curl} P_{, t}, \operatorname{Curl} P_{, t}\right\rangle, \\
& W(\nabla u, P, \operatorname{Curl} P)=\underbrace{\frac{1}{2}\left\langle\mathbb{C}_{e} \cdot \operatorname{sym}(\nabla u-P), \operatorname{sym}(\nabla u-P)\right\rangle}_{\text {anisotropic elastic - energy }}+\underbrace{\frac{1}{2}\left\langle\mathbb{C}_{\mathrm{c}} \cdot \operatorname{skew}(\nabla u-P), \text { skew }(\nabla u-P)\right\rangle}_{\text {invariant local anisotropic rotational elastic coupling }} \\
& +\underbrace{\frac{1}{2}\left\langle\mathbb{C}_{\text {micro }} \cdot \operatorname{sym} P, \operatorname{sym} P\right\rangle}_{\text {micro - self - energy }}+\underbrace{\mu \frac{L_{c}^{2}}{2}\left\langle\mathbb{L}_{\text {aniso }} \operatorname{Curl} P, \operatorname{Curl} P\right\rangle}_{\text {curvature-energy }},
\end{aligned}
$$

where

$$
\begin{cases}\mathbb{C}_{e}, \widetilde{\mathbb{C}}_{e}, \mathbb{C}_{\text {micro }}, \widetilde{\mathbb{C}}_{\text {micro }}: \operatorname{Sym}(3) \rightarrow \operatorname{Sym}(3) & \text { are dimensionless } 4^{\text {th }} \text { order tensors } \\ & \text { with } 21 \text { independent components, } \\ \mathbb{C}_{\mathrm{c}}, \widetilde{\mathbb{C}}_{\mathrm{c}}: \mathfrak{s o}(3) \rightarrow \mathfrak{s o}(3) & \text { are dimensionless } 4^{\text {th }} \text { order tensors, } \\ & \text { with } 6 \text { independent components, } \\ \mathbb{L}_{\text {aniso }}, \widetilde{\mathbb{L}}_{\text {aniso }}: \mathbb{R}^{3 \times 3} \rightarrow \mathbb{R}^{3 \times 3} & \text { are dimensionless } 4^{\text {th }} \text { order tensors, } \\ & \text { with } 45 \text { independent components, }\end{cases}
$$

the positive constants $\rho, J>0$ are the macro-inertia and micro-inertia density, $L_{c}>0$ is the characteristic length of the relaxed micromorphic model and $\mu>0$ is a parameter used for dimension compatibility of the involved terms. The limit case $L_{c} \rightarrow 0$ corresponds to considering very large specimens of a microstructured meta-material.

For simplicity, we omit the mixed terms in the form of our energies. One reason is that as long as there exist no clear mechanical interpretation of the influence of these terms on the process we want to model, we decided to keep the formulation as simple as possible. If, from some practical problems, the presence of these terms is requested, then they may be included. Another motivation, and the most important related to the present paper, is that our mathematical analysis can be extended in a straightforward manner to the case when the mixed terms are also present in the total energy. 
Compared to the classical Mindlin-Eringen model, the curvature dependence is reduced to a dependence only on the micro-dislocation tensor $\alpha:=-\operatorname{Curl} P \in \mathbb{R}^{3 \times 3}$ instead of $\gamma=\nabla P \in \mathbb{R}^{27}=\mathbb{R}^{3 \times 3 \times 3}$. Doing so, the first main advantage of the relaxed micromorphic model is that the number of constitutive coefficients is drastically reduced. A second strong point of the relaxed model is that it is possible to show that in the limit case $L_{c} \rightarrow 0$ (which corresponds to considering very large specimens of a microstructured meta-material) the mesoand micro-coefficients $\mathbb{C}_{\mathrm{e}}$ and $\mathbb{C}_{\text {micro }}$ of the relaxed model can be put in direct relation with the macroscopic stiffness of the medium via a fundamental homogenization formula, in contrast to the Eringen-Mindlin theory [17, 39], where it is impossible to obtain this kind of results, see [4, 47].

We point out another important aspect, an existence result based on the assumption that the internal energy is positive definite covers a particular situation when the energy from the classical Eringen-Mindlin micromorphic theory is positive semi-definite. Moreover, the case $\mathbb{C}_{c}=0$, i.e. the situation in which the force stress tensor is symmetric (see (2.3)), or the situation when $\mathbb{C}_{c}$ is only positive semi-definite are also covered by our analysis, and the variational setting allows to prescribe tangential boundary conditions, i.e. $P_{i}(x, t) \times n(x)=0, i=1,2,3,(x, t) \in \partial \Omega \times[0, T]$.

Only for the sake of simplicity, in the rest of the paper we assume that the constitutive coefficients $\mathbb{C}_{\mathrm{e}}, \mathbb{C}_{\text {micro }}, \mathbb{C}_{\mathrm{c}}, \mathbb{L}_{c}, \widetilde{\mathbb{C}}_{\mathrm{e}}, \widetilde{\mathbb{C}}_{\text {micro }}, \widetilde{\mathbb{C}}_{\mathrm{c}}, \widetilde{\mathbb{L}}_{c}$ are constant and they have the following symmetries

$$
\begin{array}{ll}
\left(\mathbb{C}_{\mathrm{e}}\right)_{\text {ijrs }}=\left(\mathbb{C}_{\mathrm{e}}\right)_{\text {rsij }}=\left(\mathbb{C}_{\mathrm{e}}\right)_{j i r s}, & \left(\mathbb{C}_{\mathrm{c}}\right)_{\text {ijrs }}=-\left(\mathbb{C}_{\mathrm{c}}\right)_{j i r s}=\left(\mathbb{C}_{\mathrm{c}}\right)_{\text {rsij }}, \\
\left(\mathbb{C}_{\text {micro }}\right)_{\text {ijrs }}=\left(\mathbb{C}_{\text {micro }}\right)_{\text {rsij }}=\left(\mathbb{C}_{\text {micro }}\right)_{j i r s}, & \left(\mathbb{L}_{\text {aniso }}\right)_{\text {ijrs }}=\left(\mathbb{L}_{\text {aniso }}\right)_{r s i j}, \\
\left(\widetilde{\mathbb{C}}_{\mathrm{e}}\right)_{\text {ijrs }}=\left(\widetilde{\mathbb{C}}_{\mathrm{e}}\right)_{\text {rsij }}=\left(\widetilde{\mathbb{C}}_{\mathrm{e}}\right)_{j i r s}, & \left(\widetilde{\mathbb{C}}_{\mathrm{c}}\right)_{\text {ijrs }}=-\left(\widetilde{\mathbb{C}}_{\mathrm{c}}\right)_{j i r s}=\left(\widetilde{\mathbb{C}}_{\mathrm{c}}\right)_{r s i j}, \\
\left(\widetilde{\mathbb{C}}_{\text {micro }}\right)_{\text {ijrs }}=\left(\widetilde{\mathbb{C}}_{\text {micro }}\right)_{\text {rsij }}=\left(\widetilde{\mathbb{C}}_{\text {micro }}\right)_{j i r s}, & \left(\widetilde{\mathbb{L}}_{\text {aniso }}\right)_{\text {ijrs }}=\left(\widetilde{\mathbb{L}}_{\text {aniso }}\right)_{r s i j} .
\end{array}
$$

The case of inhomogeneous media may be treated, under the supplementary assumption that the constitutive coefficients are bounded, as functions on $\Omega$.

We find, after considering the first variation of the action functional $\mathscr{A}$, that the general equations of the relaxed micromorphic model including the nonstandard inertia terms are

balance of forces :

$$
\begin{array}{r}
\rho u_{, t t}-\operatorname{Div}\left[\widetilde{\mathbb{C}}_{e} \cdot \operatorname{sym}\left(\nabla u_{, t t}-P_{, t t}\right)+\widetilde{\mathbb{C}}_{\mathrm{c}} \cdot \operatorname{skew}\left(\nabla u, t t-P_{, t t}\right)\right]= \\
\operatorname{Div}[\underbrace{\mathbb{C}_{e} \cdot \operatorname{sym}(\nabla u-P)+\mathbb{C}_{\mathrm{c}} \cdot \operatorname{skew}(\nabla u-P)}_{\sigma-\text { force stress tensor }}]+f,
\end{array}
$$

balance of moment stresses :

$$
\begin{gathered}
J P_{, t t}+\mu L_{c}^{2} \operatorname{Curl}\left[\widetilde{\mathbb{L}}_{\text {aniso }} \cdot \operatorname{Curl} P_{, t t}\right]-\widetilde{\mathbb{C}}_{e} \cdot \operatorname{sym}\left(\nabla u, t t-P_{, t t}\right)-\widetilde{\mathbb{C}}_{\mathrm{c}} \cdot \operatorname{skew}\left(\nabla u, t t-P_{, t t}\right)+\widetilde{\mathbb{C}}_{\text {micro }} \cdot \operatorname{sym} P_{, t t}= \\
-\mu L_{c}^{2} \operatorname{Curl}\left[\mathbb{L}_{\text {aniso }} \cdot \operatorname{Curl} P\right]+\mathbb{C}_{\mathrm{e}} \cdot \operatorname{sym}(\nabla u-P)+\mathbb{C}_{\mathrm{c}} \cdot \operatorname{skew}(\nabla u-P)-\mathbb{C}_{\text {micro }} \cdot \operatorname{sym} P+M .
\end{gathered}
$$

To the above system of partial differential equations, we adjoin the boundary conditions

$$
u(x, t)=0, \quad \text { and the tangential condition } \quad P_{i}(x, t) \times n(x)=0, \quad i=1,2,3, \quad(x, t) \in \partial \Omega \times[0, T],
$$

where $P_{i}, i=1,2,3$ are the rows of $P$ and the initial conditions

$$
u(x, 0)=u_{0}(x), \quad u_{, t}(x, 0)=\underline{u}_{0}(x), \quad P(x, 0)=P_{0}(x), \quad P_{, t}(x, 0)=\underline{P}_{0}(x), \quad x \in \Omega,
$$

where $u_{0}, \underline{u}_{0}, P_{0}$ and $\underline{P}_{0}$ are prescribed functions.

Regarding the tensors $\widetilde{\mathbb{C}}_{\mathrm{e}}$, and $\widetilde{\mathbb{L}}_{\text {aniso }}$, we assume that they are positive definite, i.e. there exist positive numbers $\widetilde{\mu}_{\mathrm{e}}^{m}, \widetilde{\mu}_{\mathrm{e}}^{M}>0$ (the maximum and minimum elastic moduli for $\widetilde{\mathbb{C}}_{e}$ ), $\widetilde{L}_{\mathrm{c}}^{M}, \widetilde{L}_{\mathrm{c}}^{m}>0$ (the maximum and minimum moduli for $\widetilde{\mathbb{L}}_{\text {aniso }}$ )

$$
\begin{aligned}
\widetilde{\mu}_{\mathrm{e}}^{m}\|X\|^{2} \leq\left\langle\widetilde{\mathbb{C}}_{\mathrm{e}} \cdot X, X\right\rangle \leq \widetilde{\mu}_{\mathrm{e}}^{M}\|X\|^{2} & \text { for all } \quad X \in \operatorname{Sym}(3), \\
\widetilde{L}_{c}^{m}\|X\|^{2} \leq\left\langle\widetilde{\mathbb{L}}_{\text {aniso }} \cdot X, X\right\rangle \leq \widetilde{L}_{c}^{M}\|X\|^{2} & \text { for all } \quad X \in \mathbb{R}^{3 \times 3} .
\end{aligned}
$$


We also suppose that the constitutive tensors $\widetilde{\mathbb{C}}_{c}$ and $\widetilde{\mathbb{C}}_{\text {micro }}$ are positive semi-definite, i.e

$$
\begin{aligned}
& 0 \leq\left\langle\widetilde{\mathbb{C}}_{\mathrm{c}} \cdot X, X\right\rangle \quad \text { for all } \quad X \in \mathfrak{s o}(3), \\
& 0 \leq\left\langle\widetilde{\mathbb{C}}_{\text {micro }} \cdot X, X\right\rangle \quad \text { for all } X \in \operatorname{Sym}(3) \text {, }
\end{aligned}
$$

which means that we do not exclude the possibility that these constitutive tensors vanish.

Since the media under consideration is homogeneous, the quadratic forms defined by the fourth order elasticity tensors $\mathbb{C}_{e}, \mathbb{C}_{\mathrm{c}}, \mathbb{C}_{\text {micro }}$ and $\mathbb{L}_{\text {aniso }}$ and by the tensors $\widetilde{\mathbb{C}}_{\mathrm{c}}$ and $\widetilde{\mathbb{C}}_{\text {micro }}$, respectively, are bounded, i.e. there exist $\mu_{\mathrm{e}}^{M} \geq 0, \mu_{\mathrm{c}}^{M} \geq 0, \mu_{\text {micro }}^{M} \geq 0, L_{\mathrm{c}}^{M} \geq 0, \widetilde{\mu}_{\mathrm{c}}^{M} \geq 0$ and $\widetilde{\mu}_{\text {micro }}^{M} \geq 0$ such that

$$
\begin{array}{rll}
\left\langle\mathbb{C}_{\mathrm{e}} \cdot X, X\right\rangle \leq \mu_{\mathrm{e}}^{M}\|X\|^{2} & \text { for all } & X \in \operatorname{Sym}(3), \\
\left\langle\mathbb{C}_{\mathrm{c}} \cdot X, X\right\rangle \leq \mu_{\mathrm{c}}^{M}\|X\|^{2} & \text { for all } & X \in \mathfrak{s o}(3), \\
\left\langle\mathbb{L}_{\text {aniso }} \cdot X, X\right\rangle \leq L_{c}^{M}\|X\|^{2} & \text { for all } & X \in \mathbb{R}^{3 \times 3}, \\
\left\langle\mathbb{C}_{\text {micro }} \cdot X, X\right\rangle \leq \mu_{\text {micro }}^{M}\|X\|^{2} & \text { for all } & X \in \operatorname{Sym}(3), \\
\left\langle\widetilde{\mathbb{C}}_{\mathrm{c}} \cdot X, X\right\rangle \leq \widetilde{\mu}_{\mathrm{c}}^{M}\|X\|^{2} & \text { for all } & X \in \mathfrak{s o}(3), \\
\left\langle\widetilde{\mathbb{C}}_{\text {micro }} \cdot X, X\right\rangle \leq \widetilde{\mu}_{\text {micro }}^{M}\|X\|^{2} & \text { for all } & X \in \operatorname{Sym}(3) .
\end{array}
$$

In the rest of this paper we denote tensors having this property as bounded tensors. In our assumptions the elastic constitutive tensors may be negative definite or positive semi-definite, or they may even vanish.

In the next subsection, we will prove that the above model is well-posed.

\subsection{Existence of the solution}

We introduce two bilinear forms $\mathcal{W}_{1}, \mathcal{W}_{2}:\left(H_{0}^{1}(\Omega) \times H_{0}(\operatorname{Curl} ; \Omega)\right) \times\left(H_{0}^{1}(\Omega) \times H_{0}(\operatorname{Curl} ; \Omega)\right) \rightarrow \mathbb{R}$

$$
\begin{aligned}
\mathcal{W}_{1}((u, P),(\varphi, \Phi))=\int_{\Omega} & \left(\rho\langle u, \varphi\rangle+J\langle P, \Phi\rangle+\left\langle\widetilde{\mathbb{C}}_{\mathrm{e}} \cdot \operatorname{sym}(\nabla u-P), \operatorname{sym}(\nabla \varphi-\Phi)\right\rangle\right. \\
& +\left\langle\widetilde{\mathbb{C}}_{\mathrm{c}} \cdot \operatorname{skew}(\nabla u-P), \operatorname{skew}(\nabla \varphi-\Phi)\right\rangle+\left\langle\widetilde{\mathbb{C}}_{\text {micro }} \cdot \operatorname{sym} P, \operatorname{sym} \Phi\right\rangle \\
& \left.+\mu L_{c}^{2}\left\langle\widetilde{\mathbb{L}}_{\text {aniso }} \cdot \operatorname{Curl} P, \operatorname{Curl} \Phi\right\rangle\right) d v \\
\mathcal{W}_{2}((u, P),(\varphi, \Phi))=\int_{\Omega}( & \left\langle\mathbb{C}_{\mathrm{e}} \cdot \operatorname{sym}(\nabla u-P), \operatorname{sym}(\nabla \varphi-\Phi)\right\rangle+\left\langle\mathbb{C}_{\mathrm{c}} \cdot \operatorname{skew}(\nabla u-P), \operatorname{skew}(\nabla \varphi-\Phi)\right\rangle \\
& \left.+\left\langle\mathbb{C}_{\text {micro }} \cdot \operatorname{sym} P, \operatorname{sym} \Phi\right\rangle+\mu L_{c}^{2}\left\langle\mathbb{L}_{\text {aniso }} \cdot \operatorname{Curl} P, \operatorname{Curl} \Phi\right\rangle\right) d v
\end{aligned}
$$

and for each $(f, M) \in H^{-1}(\Omega) \times\left(H_{0}(\mathrm{Curl} ; \Omega)\right)^{*}$ we consider the linear operator $l^{(f, M)} \in H^{-1}(\Omega) \times\left(H_{0}(\operatorname{Curl} ; \Omega)\right)^{*}$ defined by

$$
l^{(f, M)}: H_{0}^{1}(\Omega) \times H_{0}(\mathrm{Curl} ; \Omega) \rightarrow \mathbb{R}, \quad l^{(f, M)}(\varphi, \Phi)=\langle f, \varphi\rangle_{H^{-1}(\Omega), H_{0}^{1}(\Omega)}+\langle M, \Phi\rangle_{\left(H_{0}(\mathrm{Curl} ; \Omega)\right)^{*}, H_{0}(\mathrm{Curl} ; \Omega)},
$$

where $H^{-1}(\Omega)$ and $\left(H_{0}(\mathrm{Curl} ; \Omega)\right)^{*}$ are the dual spaces of $H_{0}^{1}(\Omega)$ and $H_{0}(\mathrm{Curl} ; \Omega)$, respectively. We equip the product space $H_{0}^{1}(\Omega) \times H_{0}(\mathrm{Curl} ; \Omega)$ with the norm

$$
\|(u, P)\|_{H_{0}^{1}(\Omega) \times H_{0}(\operatorname{Curl} ; \Omega)}=\left(\|u\|_{H_{0}^{1}(\Omega)}^{2}+\|P\|_{H_{0}(\operatorname{Curl} ; \Omega)}^{2}\right)^{1 / 2} .
$$

For every $(f, M) \in C\left([0, T] ; H^{-1}(\Omega) \times\left(H_{0}(\mathrm{Curl} ; \Omega)\right)^{*}\right.$, the pair $(u, P) \in C^{2}\left([0, T] ; H_{0}^{1}(\Omega) \times H_{0}(\mathrm{Curl} ; \Omega)\right)$ is a weak solution of the problem $2.3-2.5$ provided

$$
\mathcal{W}_{1}\left(\left(u, t t(t), P_{, t t}(t)\right),(\varphi, \Phi)\right)+\mathcal{W}_{2}((u(t), P(t)),(\varphi, \Phi))=l^{(f(t), M(t))}(\varphi, \Phi)
$$

for each $(\varphi, \Phi) \in H_{0}^{1}(\Omega) \times H_{0}(\mathrm{Curl} ; \Omega)$ and for all $t \in[0, T]$, and it satisfies

$$
u(x, 0)=u_{0}(x), \quad u_{, t}(x, 0)=\underline{u}_{0}(x), \quad P(x, 0)=P_{0}(x), \quad P_{, t}(x, 0)=\underline{P}_{0}(x), \quad x \in \Omega .
$$


The proof that any classical solution is a weak solution follows using analogue calculations as in 45.

In order to prove the existence and uniqueness of a weak solution of problem $(2.3)$, we follow the strategy proposed in [9] and we first prove the following lemma.

Lemma 2.1. Assume that

i) the constitutive tensors satisfy the symmetry relations 2.2 , respectively,

ii) $\mathbb{C}_{\mathrm{e}}, \mathbb{C}_{\text {micro }}, \mathbb{C}_{\mathrm{c}}$ and $\mathbb{L}_{\text {aniso }}$ are bounded tensors,

iii) $\widetilde{\mathbb{C}}_{\mathrm{e}}$ and $\widetilde{\mathbb{L}}_{\text {aniso }}$ are positive definite tensors,

iv) $\widetilde{\mathbb{C}}_{\text {micro }}$ and $\widetilde{\mathbb{C}}_{\mathrm{c}}$ are positive semi-definite tensors,

v) $(f, M) \in C\left([0, T] ; H^{-1}(\Omega) \times\left(H_{0}(\mathrm{Curl} ; \Omega)\right)^{*}\right)$,

vi) $\left(u_{0}, P_{0}\right),\left(\underline{u}_{0}, \underline{P}_{0}\right) \in H_{0}^{1}(\Omega) \times H_{0}(\operatorname{Curl} ; \Omega)$,

vii) $\rho, J, L_{c}, \mu>0$,

then, for all $(v, Q) \in C\left([0, T] ; H_{0}^{1}(\Omega) \times H_{0}(\mathrm{Curl} ; \Omega)\right)$, there exists a unique function $(u, P) \in C^{2}\left([0, T] ; H_{0}^{1}(\Omega) \times\right.$ $\left.H_{0}(\mathrm{Curl} ; \Omega)\right)$ such that

$$
\mathcal{W}_{1}\left(\left(u_{, t t}(t), P_{, t t}(t)\right),(\varphi, \Phi)\right)=-\mathcal{W}_{2}((v(t), Q(t)),(\varphi, \Phi))+l^{(f(t), M(t))}(\varphi, \Phi),
$$

for all $(\varphi, \Phi) \in H_{0}^{1}(\Omega) \times H_{0}(\operatorname{Curl} ; \Omega)$ and for all $t \in[0, T]$, and it satisfies

$$
u(x, 0)=u_{0}(x), \quad u_{, t}(x, 0)=\underline{u}_{0}(x), \quad P(x, 0)=P_{0}(x), \quad P_{, t}(x, 0)=\underline{P}_{0}(x), \quad x \in \Omega .
$$

Proof. Let us consider a fixed time $t \in[0, T]$. From the Cauchy-Schwarz inequality and Poincaré inequality, and since the constitutive coefficients satisfy $(2.8)$, we find that for fixed $(w, R) \in H_{0}^{1}(\Omega) \times H_{0}(\mathrm{Curl} ; \Omega)$ the map $\mathcal{W}_{2}((w, R), \cdot): H_{0}^{1}(\Omega) \times H_{0}(\mathrm{Curl} ; \Omega) \rightarrow \mathbb{R}$ is bounded, since

$$
\begin{aligned}
& \|\operatorname{sym}(\nabla \varphi-\Phi)\|^{2} \leq 2\left(\|\operatorname{sym} \nabla \varphi\|^{2}+\|\operatorname{sym} \Phi\|^{2}\right), \quad\|\operatorname{sym} \nabla \varphi\|^{2} \leq\|\nabla \varphi\|^{2}, \quad\|\operatorname{sym} \Phi\|^{2} \leq\|\Phi\|^{2}, \\
& \|\operatorname{skew}(\nabla \varphi-\Phi)\|^{2} \leq 2\left(\|\operatorname{skew} \nabla \varphi\|^{2}+\|\operatorname{skew} \Phi\|^{2}\right), \quad\|\operatorname{skew} \nabla \varphi\|^{2} \leq\|\nabla \varphi\|^{2}, \quad\|\operatorname{skew} \Phi\|^{2} \leq\|\Phi\|^{2},
\end{aligned}
$$

for all $\varphi \in H_{0}^{1}(\Omega)$ and for all $\Phi \in L^{2}(\Omega)$. Similarly it follows that $\mathcal{W}_{1}$ is bounded. On the other hand

$$
\begin{aligned}
& \mathcal{W}_{1}((\varphi, \Phi),(\varphi, \Phi))=\int_{\Omega}( \rho\|\varphi\|^{2}+J\|\Phi\|^{2}+\left\langle\widetilde{\mathbb{C}}_{\mathrm{e}} \cdot \operatorname{sym}(\nabla \varphi-\Phi), \operatorname{sym}(\nabla \varphi-\Phi)\right\rangle \\
&+\left\langle\widetilde{\mathbb{C}}_{\mathrm{c}} \cdot \operatorname{skew}(\nabla \varphi-\Phi), \operatorname{skew}(\nabla \varphi-\Phi)\right\rangle+\left\langle\widetilde{\mathbb{C}}_{\text {micro }} \cdot \operatorname{sym} \Phi, \operatorname{sym} \Phi\right\rangle \\
&\left.\quad+\mu L_{c}^{2}\left\langle\widetilde{\mathbb{L}}_{\text {aniso }} \cdot \operatorname{Curl} \Phi, \operatorname{Curl} \Phi\right\rangle\right) d v \\
& \geq \int_{\Omega}\left(\rho\|\varphi\|^{2}+J\|\Phi\|^{2}+\left\langle\widetilde{\mathbb{C}}_{\mathrm{e}} \cdot \operatorname{sym}(\nabla \varphi-\Phi), \operatorname{sym}(\nabla \varphi-\Phi)\right\rangle+\mu L_{c}^{2}\left\langle\widetilde{\mathbb{L}}_{\text {aniso }} \cdot \operatorname{Curl} \Phi, \operatorname{Curl} \Phi\right\rangle\right) d v \\
& \geq \int_{\Omega}\left(\rho\|\varphi\|^{2}+\frac{J}{2}\|\Phi\|^{2}+\frac{J}{2}\|\operatorname{sym} \Phi\|^{2}+\widetilde{\mu}_{\mathrm{e}}^{m}\|\operatorname{sym}(\nabla \varphi-\Phi)\|^{2}+\mu L_{c}^{2} \widetilde{L}_{\mathrm{c}}^{m}\|\operatorname{Curl} \Phi\|^{2}\right) d v
\end{aligned}
$$

for all $(\varphi, \Phi) \in H_{0}^{1}(\Omega) \times H_{0}(\mathrm{Curl} ; \Omega)$, since, if it is present, the tensor $\widetilde{\mathbb{C}}_{\mathrm{c}}$ and $\widetilde{\mathbb{C}}_{\text {micro }}$ are positive semi-definite, while $\widetilde{\mathbb{L}}_{\text {aniso }}$ and $\widetilde{\mathbb{C}}_{c}$ are positive definite.

Since

$$
\|\operatorname{sym} \nabla \varphi\|^{2} \leq\|\operatorname{sym}(\nabla \varphi-\Phi)\|^{2}+\|\operatorname{sym} \Phi\|^{2}
$$

for all $\varphi \in \mathrm{H}^{1}(\Omega)$ and $\Phi \in L^{2}(\Omega)$, we have that there exists $c>0$ such that

$$
\begin{aligned}
\mathcal{W}_{1}((\varphi, \Phi),(\varphi, \Phi)) & \geq c \int_{\Omega}\left(\|\varphi\|^{2}+\|\Phi\|^{2}+\|\operatorname{sym} \nabla \varphi\|^{2}+\|\operatorname{Curl} \Phi\|^{2}\right) d v \\
& \geq c \int_{\Omega}\left(\|\operatorname{sym} \nabla \varphi\|^{2}+\|\Phi\|^{2}+\|\operatorname{Curl} \Phi\|^{2}\right) d v
\end{aligned}
$$


for all $(\varphi, \Phi) \in H_{0}^{1}(\Omega) \times H_{0}(\mathrm{Curl} ; \Omega)$. Hence, the coercivity of $\mathcal{W}_{1}$ is assured by the coercivity of the quadratic form from the right-hand side of the above inequality, which is a direct consequence of the classical Korn inequality 40 .

Since the linear operator $l^{(f(t), M(t))}$ is bounded for a fixed time $t$ considered in the beginning of the proof, using the Lax-Milgram theorem we obtain the existence of a unique solution $\left(u^{*}(t), P^{*}(t)\right) \in H_{0}^{1}(\Omega) \times H_{0}(\mathrm{Curl} ; \Omega)$ of the equation

$$
\mathcal{W}_{1}\left(\left(u^{*}(t), P^{*}(t)\right),(\varphi, \Phi)\right)=-\mathcal{W}_{2}((v(t), Q(t)),(\varphi, \Phi))+l^{(f(t), M(t))}(\varphi, \Phi)
$$

for all $(\varphi, \Phi) \in H_{0}^{1}(\Omega) \times H_{0}(\operatorname{Curl} ; \Omega)$. From standard arguments it follows that if $(f, M) \in C\left([0, T] ; H^{-1}(\Omega) \times\right.$ $\left.\left(H_{0}(\mathrm{Curl} ; \Omega)\right)^{*}\right)$ and $(v, Q) \in C\left([0, T] ; H_{0}^{1}(\Omega) \times H_{0}(\mathrm{Curl} ; \Omega)\right)$, then the solution $\left(u^{*}, P^{*}\right)$ of (2.16) belongs to $C\left([0, T] ; H_{0}^{1}(\Omega) \times H_{0}(\mathrm{Curl} ; \Omega)\right)$. Indeed, the difference of the solutions of 2.16$)$ which correspond to two times $t_{1}, t_{2} \in[0, T]$ satisfies

$$
\begin{aligned}
\mathcal{W}_{1}\left(\left(u^{*}\left(t_{1}\right)-u^{*}\left(t_{2}\right),\right.\right. & \left.\left.P^{*}\left(t_{1}\right)-P^{*}\left(t_{2}\right)\right),(\varphi, \Phi)\right)=-\mathcal{W}_{2}\left(\left(v\left(t_{1}\right)-v\left(t_{2}\right), Q\left(t_{1}\right)-Q\left(t_{2}\right)\right),(\varphi, \Phi)\right) \\
& +\left\langle f\left(t_{1}\right)-f\left(t_{2}\right), \varphi\right\rangle_{H^{-1}(\Omega), H_{0}^{1}(\Omega)}+\left\langle M\left(t_{1}\right)-M\left(t_{2}\right), \Phi\right\rangle_{H_{0}^{*}(\mathrm{Curl} ; \Omega), H_{0}(\mathrm{Curl} ; \Omega)}
\end{aligned}
$$

for all $(\varphi, \Phi) \in H_{0}^{1}(\Omega) \times H_{0}(\operatorname{Curl} ; \Omega)$. Using the coercivity of $\mathcal{W}_{1}$ and the boundedness of $\mathcal{W}_{2}$ we obtain that

$$
\begin{aligned}
\left\|u^{*}\left(t_{1}\right)-u^{*}\left(t_{2}\right)\right\|_{H_{0}^{1}(\Omega)}^{2}+ & \left\|P^{*}\left(t_{1}\right)-P^{*}\left(t_{2}\right)\right\|_{H_{0}(\operatorname{Curl} ; \Omega)}^{2} \\
\leq & c\left(\left\|u^{*}\left(t_{1}\right)-u^{*}\left(t_{2}\right)\right\|_{H_{0}^{1}(\Omega)}^{2}+\left\|P^{*}\left(t_{1}\right)-P^{*}\left(t_{2}\right)\right\|_{H_{0}(\mathrm{Curl} ; \Omega)}^{2}\right)^{1 / 2} \\
& {\left[\left(\left\|v\left(t_{1}\right)-v\left(t_{2}\right)\right\|_{H_{0}^{1}(\Omega)}^{2}+\left\|Q\left(t_{1}\right)-Q\left(t_{2}\right)\right\|_{H_{0}(\mathrm{Curl} ; \Omega)}^{2}\right)^{1 / 2}\right.} \\
\quad & \left.\quad\left(\left\|f\left(t_{1}\right)-f\left(t_{2}\right)\right\|_{H^{-1}(\Omega)}^{2}+\left\|M\left(t_{1}\right)-M\left(t_{2}\right)\right\|_{H^{*}(\mathrm{Cur} ; \Omega)}^{2}\right)^{1 / 2}\right],
\end{aligned}
$$

where $c$ is a positive constant. Hence, there is a positive constant $c>0$ such that

$$
\begin{aligned}
& \left\|u^{*}\left(t_{1}\right)-u^{*}\left(t_{2}\right)\right\|_{H_{0}^{1}(\Omega)}^{2}+\left\|P^{*}\left(t_{1}\right)-P^{*}\left(t_{2}\right)\right\|_{H_{0}(\mathrm{Curl} ; \Omega)}^{2} \\
& \leq c\left[\left\|v\left(t_{1}\right)-v\left(t_{2}\right)\right\|_{H_{0}^{1}(\Omega)}^{2}+\left\|Q\left(t_{1}\right)-Q\left(t_{2}\right)\right\|_{H_{0}(\mathrm{Curl} ; \Omega)}^{2}+\left\|f\left(t_{1}\right)-f\left(t_{2}\right)\right\|_{H^{-1}(\Omega)}^{2}+\left\|M\left(t_{1}\right)-M\left(t_{2}\right)\right\|_{H^{*}(\mathrm{Curl} ; \Omega)}^{2}\right],
\end{aligned}
$$

which proves the continuity.

Now, the unique solution $(u, P) \in C^{2}\left([0, T] ; H_{0}^{1}(\Omega) \times H_{0}(\mathrm{Curl} ; \Omega)\right)$ of the problem defined by 2.12 and 2.13 will be

$$
u(t)=u_{0}+\int_{0}^{t}\left(\underline{u}_{0}+\int_{0}^{s} u^{*}(\xi) d \xi\right) d s, \quad P(t)=P_{0}+\int_{0}^{t}\left(\underline{P}_{0}+\int_{0}^{s} P^{*}(\xi) d \xi\right) d s
$$

for all $t \in[0, T]$ and the proof is complete.

Theorem 2.2. Assume that

i) the constitutive tensors satisfy the symmetry relations 2.2 , respectively,

ii) $\mathbb{C}_{\mathrm{e}}, \mathbb{C}_{\text {micro }}, \mathbb{C}_{\mathrm{c}}$ and $\mathbb{L}_{\text {aniso }}$ are bounded tensors,

iii) $\widetilde{\mathbb{C}}_{\mathrm{e}}$ and $\widetilde{\mathbb{L}}_{\text {aniso }}$ are positive definite tensors,

iv) $\widetilde{\mathbb{C}}_{\text {micro }}$ and $\widetilde{\mathbb{C}}_{\mathrm{c}}$ are positive semi-definite tensors,

v) $(f, M) \in C\left([0, T] ; H^{-1}(\Omega) \times\left(H_{0}(\mathrm{Curl} ; \Omega)\right)^{*}\right)$,

vi) $\left(u_{0}, P_{0}\right),\left(\underline{u}_{0}, \underline{P}_{0}\right) \in H_{0}^{1}(\Omega) \times H_{0}(\mathrm{Curl} ; \Omega)$,

vii) $\rho, J, L_{c}, \mu>0$,

then there exists a unique solution $(u, P) \in C^{2}\left([0, T] ; H_{0}^{1}(\Omega) \times H_{0}(\mathrm{Curl} ; \Omega)\right)$ of the problem defined by 2.10$)$ and (2.11). 
Proof. To prove this theorem, we will use the Banach fixed-point theorem for the mapping

$$
\mathcal{L}: C\left([0, \delta] ; H_{0}^{1}(\Omega) \times H_{0}(\mathrm{Curl} ; \Omega)\right) \rightarrow C^{2}\left([0, \delta] ; H_{0}^{1}(\Omega) \times H_{0}(\mathrm{Curl} ; \Omega)\right) \subset C\left([0, \delta] ; H_{0}^{1}(\Omega) \times H_{0}(\mathrm{Curl} ; \Omega)\right)
$$

which, for fixed $(f, M) \in C\left([0, \delta] ; H^{-1}(\Omega) \times\left(H_{0}(\operatorname{Curl} ; \Omega)\right)^{*}\right),\left(u_{0}, P_{0}\right),\left(\underline{u}_{0}, \underline{P}_{0}\right) \in H_{0}^{1}(\Omega) \times H_{0}(\operatorname{Curl} ; \Omega)$, maps each $(v, Q) \in C\left([0, \delta] ; H_{0}^{1}(\Omega) \times H_{0}(\operatorname{Curl} ; \Omega)\right)$ to the solution of the corresponding problem defined by 2.12 and (2.13), where $\delta>0$ will be suitably chosen.

Let us consider $\left(v^{(1)}, Q^{(1)}\right),\left(v^{(2)}, Q^{(2)}\right) \in C\left([0, \delta] ; H_{0}^{1}(\Omega) \times H_{0}(\mathrm{Curl} ; \Omega)\right)$ and their corresponding solutions $\left(u^{(1)}, P^{(1)}\right),\left(u^{(2)}, P^{(2)}\right) \in C^{2}\left([0, \delta] ; H_{0}^{1}(\Omega) \times H_{0}(\mathrm{Curl} ; \Omega)\right)$ of the related problems defined by 2.12) and 2.13), i.e.

$$
u^{(\alpha)}(t)=u_{0}+\int_{0}^{t}\left(\underline{u}_{0}+\int_{0}^{s} u^{(\alpha) *}(\xi) d \xi\right) d s, \quad P^{(\alpha)}(t)=P_{0}+\int_{0}^{t}\left(\underline{P}_{0}+\int_{0}^{s} P^{(\alpha) *}(\xi) d \xi\right) d s, \quad \alpha=1,2,
$$

for all $t \in[0, \delta]$, where $\left(u^{(\alpha) *}, P^{(\alpha) *}\right) \in C\left([0, \delta] ; H_{0}^{1}(\Omega) \times H_{0}(\mathrm{Curl} ; \Omega)\right)$ is the unique solution of the equation:

$$
\mathcal{W}_{1}\left(\left(u^{(\alpha) *}(t), P^{(\alpha) *}(t)\right),(\varphi, \Phi)\right)=-\mathcal{W}_{2}((v(t), Q(t)),(\varphi, \Phi))+l^{(f(t), M(t))}(\varphi, \Phi)
$$

for all $(\varphi, \Phi) \in H_{0}^{1}(\Omega) \times H_{0}(\mathrm{Curl} ; \Omega)$ and for all $t \in[0, \delta]$.

Then, because the solutions correspond to the same initial conditions and forces, we have

$$
\begin{aligned}
\max _{t \in[0, \delta]}\left(\| u^{(1)}(t)\right. & \left.-u^{(2)}(t)\left\|_{H_{0}^{1}(\Omega)}+\right\| P^{(1)}(t)-P^{(2)}(t) \|_{H_{0}(\mathrm{Curl} ; \Omega)}\right) \\
& \leq \max _{t \in[0, \delta]} \int_{0}^{t} \int_{0}^{s}\left(\left\|u^{(1) *}(\xi)-u^{(2) *}(\xi)\right\|_{H_{0}^{1}(\Omega)}+\left\|P^{(1) *}(\xi)-P^{(2) *}(\xi)\right\|_{H_{0}(\mathrm{Curl} ; \Omega)}\right) d \xi d s
\end{aligned}
$$

Since the problem is linear, $\left(u^{(1) *}-u^{(2) *}, P^{(1) *}-P^{(2) *}\right) \in C\left([0, \delta] ; H_{0}^{1}(\Omega) \times H_{0}(\mathrm{Curl} ; \Omega)\right)$ satisfies

$$
\mathcal{W}_{1}\left(\left(u^{(1) *}(t)-u^{(2) *}(t), P^{(1) *}(t)-P^{(2) *}(t)\right),(\varphi, \Phi)\right)=-\mathcal{W}_{2}\left(\left(v^{(1)}(t)-v^{(2)}(t), Q^{(1)}(t)-Q^{(2)}(t)\right),(\varphi, \Phi)\right)
$$

for all $(\varphi, \Phi) \in H_{0}^{1}(\Omega) \times H_{0}(\operatorname{Curl} ; \Omega)$ and for all $t \in[0, \delta]$. The coercivity of $\mathcal{W}_{1}$ and the boundedness of $\mathcal{W}_{2}$ lead us to

$$
\begin{aligned}
\| u^{(1) *}(t) & -u^{(2) *}(t)\left\|_{H_{0}^{1}(\Omega)}^{2}+\right\| P^{(1) *}(t)-P^{(2) *}(t) \|_{H_{0}(\mathrm{Curl} ; \Omega)}^{2} \\
& \leq c\left(\left\|v^{(1)}(t)-v^{(2)}(t)\right\|_{H_{0}^{1}(\Omega)}^{2}+\left\|Q^{(1)}(t)-Q^{(2)}(t)\right\|_{H_{0}(\mathrm{Curl} ; \Omega)}^{2}\right),
\end{aligned}
$$

where $c$ is a positive constant. But

$$
\begin{aligned}
\frac{1}{2}\left(\left\|u^{(1) *}(t)-u^{(2) *}(t)\right\|_{H_{0}^{1}(\Omega)}\right. & \left.+\left\|P^{(1) *}(t)-P^{(2) *}(t)\right\|_{H_{0}(\mathrm{Curl} ; \Omega)}\right)^{2} \\
& \leq\left\|u^{(1) *}(t)-u^{(2) *}(t)\right\|_{H_{0}^{1}(\Omega)}^{2}+\left\|P^{(1) *}(t)-P^{(2) *}(t)\right\|_{H_{0}(\mathrm{Curl} ; \Omega)}^{2},
\end{aligned}
$$

and therefore we deduce that

$$
\begin{aligned}
\left\|u^{(1) *}(t)-u^{(2) *}(t)\right\|_{H_{0}^{1}(\Omega)} & +\left\|P^{(1) *}(t)-P^{(2) *}(t)\right\|_{H_{0}(\operatorname{Curl} ; \Omega)} \\
& \leq \sqrt{2 c}\left(\left\|v^{(1)}(t)-v^{(2)}(t)\right\|_{H_{0}^{1}(\Omega)}^{2}+\left\|Q^{(1)}(t)-Q^{(2)}(t)\right\|_{H_{0}(\mathrm{Curl} ; \Omega)}^{2}\right)^{1 / 2} .
\end{aligned}
$$

Hence, we obtain

$$
\begin{aligned}
\max _{t \in[0, \delta]}\left(\| u^{(1)}(t)\right. & \left.-u^{(2)}(t)\left\|_{H_{0}^{1}(\Omega)}^{2}+\right\| P^{(1)}(t)-P^{(2)}(t) \|_{H_{0}(\operatorname{Curl} ; \Omega)}^{2}\right)^{1 / 2} \\
& \leq \max _{t \in[0, \delta]}\left(\left\|u^{(1)}(t)-u^{(2)}(t)\right\|_{H_{0}^{1}(\Omega)}+\left\|P^{(1)}(t)-P^{(2)}(t)\right\|_{H_{0}(\mathrm{Curl} ; \Omega)}\right) \\
& \leq \sqrt{2 c} \max _{t \in[0, \delta]} \int_{0}^{t} \int_{0}^{s}\left(\left\|v^{(1)}(\xi)-v^{(2)}(\xi)\right\|_{H_{0}^{1}(\Omega)}^{2}+\left\|Q^{(1)}(\xi)-Q^{(2)}(\xi)\right\|_{H_{0}(\mathrm{Cur} ; \Omega)}^{2}\right)^{1 / 2} d \xi d s \\
& \leq \delta^{2} \sqrt{2 c} \max _{t \in[0, \delta]}\left(\left\|v^{(1)}(t)-v^{(2)}(t)\right\|_{H_{0}^{1}(\Omega)}^{2}+\left\|Q^{(1)}(t)-Q^{(2)}(t)\right\|_{H_{0}(\mathrm{Curl} ; \Omega)}^{2}\right)^{1 / 2} .
\end{aligned}
$$


Therefore

$$
\begin{aligned}
\max _{t \in[0, \delta]}\left(\| u^{(1)}(t)\right. & \left.-u^{(2)}(t)\left\|_{H_{0}^{1}(\Omega)}^{2}+\right\| P^{(1)}(t)-P^{(2)}(t) \|_{H_{0}(\mathrm{Cur} ; \Omega)}^{2}\right)^{1 / 2} \\
& \leq \delta^{2} c \max _{t \in[0, \delta]}\left(\left\|v^{(1)}(t)-v^{(2)}(t)\right\|_{H_{0}^{1}(\Omega)}^{2}+\left\|Q^{(1)}(t)-Q^{(2)}(t)\right\|_{H_{0}(\mathrm{Curl} ; \Omega)}^{2}\right)^{1 / 2},
\end{aligned}
$$

where $c$ is positive constant which is independent of time and also of the initial condition.

Hence, $\mathcal{L}$ is a contraction on $C\left([0, \delta] ; H_{0}^{1}(\Omega) \times H_{0}(\mathrm{Curl} ; \Omega)\right)$ for $\delta=\frac{1}{2 \sqrt{c}}$ with $c$ taken from the above estimate. Hence, for $\delta=\frac{1}{2 \sqrt{c}}$, there exists a unique function $(u, P) \in C\left([0, \delta] ; H_{0}^{1}(\Omega) \times H_{0}(\mathrm{Curl} ; \Omega)\right)$ such that

$$
(u, P)=\mathcal{L} .(u, P) \in C^{2}\left([0, \delta] ; H_{0}^{1}(\Omega) \times H_{0}(\operatorname{Curl} ; \Omega)\right) .
$$

Therefore, there exists a unique $(u, P) \in C^{2}\left([0, \delta] ; H_{0}^{1}(\Omega) \times H_{0}(\mathrm{Curl} ; \Omega)\right)$ solution of the problem

$$
\mathcal{W}_{1}\left(\left(u, t t(t), P_{, t t}(t),(\varphi, \Phi)\right)=-\mathcal{W}_{2}\left((u(t), P(t),(\varphi, \Phi))+l^{(f(t), M(t)}(\varphi, \Phi)\right.\right.
$$

for all $(\varphi, \Phi) \in H_{0}^{1}(\Omega) \times H_{0}(\operatorname{Curl} ; \Omega)$ and for all $t \in[0, \delta]$, which satisfies

$$
u(x, 0)=u_{0}(x), \quad u_{, t}(x, 0)=\underline{u}_{0}(x), \quad P(x, 0)=P_{0}(x), \quad P_{, t}(x, 0)=\underline{P}_{0}(x), \quad x \in \Omega
$$

for all $t \in[0, \delta]$.

By repeating the above analysis, since the positive constant $c$ in 2.26 does not depend on the initial data, we argue that there exists a unique solution $(\widetilde{u}, \widetilde{P}) \in C^{2}\left([\delta, 2 \delta] ; H_{0}^{1}(\Omega) \times H_{0}(\mathrm{Curl} ; \Omega)\right)$ of the problem

$$
\mathcal{W}_{1}\left(\left(\widetilde{u}_{, t t}(t), \widetilde{P}_{, t t}(t),(\varphi, \Phi)\right)=-\mathcal{W}_{2}\left((\widetilde{u}(t), \widetilde{P}(t),(\varphi, \Phi))+l^{(f(t), M(t)}(\varphi, \Phi)\right.\right.
$$

for all $(\varphi, \Phi) \in H_{0}^{1}(\Omega) \times H_{0}(\mathrm{Curl} ; \Omega)$ and for all $t \in[\delta, 2 \delta]$, which satisfies

$$
\widetilde{u}(x, \delta)=u(x, \delta), \quad \widetilde{u}_{, t}(x, \delta)=u_{, t}(x, \delta), \quad \widetilde{P}(x, \delta)=P(x, \delta), \quad \widetilde{P}_{, t}(x, \delta)=P_{, t}(x, \delta), \quad x \in \Omega .
$$

Putting together the solution on $[0, \delta]$ and on $[\delta, 2 \delta]$ we obtain a solution $(u, P) \in C^{2}\left([0,2 \delta] ; H_{0}^{1}(\Omega) \times\right.$ $\left.H_{0}(\operatorname{Curl} ; \Omega)\right)$ of the initial problem of the problem defined by $(2.10)$ and $(2.11)$, due to the fact that $\left(u_{, t t}, P_{, t t}\right) \in$ $C\left([0,2 \delta] ; H_{0}^{1}(\Omega) \times H_{0}(\mathrm{Curl} ; \Omega)\right)$. The continuity of $\left(u_{, t t}, P_{, t t}\right)$ follows from the continuity on $[0, \delta]$ and $[\delta, 2 \delta]$, respectively, of the functions $\left(u^{*}, P^{*}\right)$ defining the function $(u, P)$ through expressions 2.19].

Hence, due to similar iterations, we may extend the already constructed solution to the interval $[0, T]$ by considering a large enough step $n>2 T \sqrt{c}$, where $c$ is the constant from inequality 2.26 .

\section{Remark 2.1.}

i) We note that the application of the Banach fixed-point theorem on small intervals and to glue the fixed points together may be avoided by using an exponential weight in the maximum norm, as in the classical proof of the Picard-Lindelöf theorem. For a similar observation in this context and for other remarks regarding the presence of time derivatives in models one may consult [55].

ii) While in proving the existence of solution for the relaxed model without novel inertia terms (see [24, 45]) the requirement that $\mathbb{C}_{\text {micro }}$ is positive definite was essential, in the relaxed model which includes new inertia terms, the existence result is still valid when $\widetilde{\mathbb{C}}_{\text {micro }}$ is only positive semi-definite and $\mathbb{C}_{\text {micro }}$ is only bounded.

\section{On the existence for a simplified model}

The aim of this section is to investigate if the problem remains well-posed when we take into account the following simplified expression for the kinetic energy

$$
\begin{aligned}
K\left(u_{, t}, P_{, t}, \nabla u_{, t}, \operatorname{Curl} P_{, t}\right)= & \frac{1}{2}\left\langle\rho u_{, t}, u_{, t}\right\rangle+\frac{1}{2}\left\langle\widetilde{\mathbb{C}}_{e} \operatorname{sym}\left(\nabla u_{, t}-P_{, t}\right), \operatorname{sym}\left(\nabla u_{, t}-P_{, t}\right)\right\rangle \\
& +\frac{1}{2}\left\langle\widetilde{\mathbb{C}}_{c} \cdot \operatorname{skew}\left(\nabla u_{, t}-P_{, t}\right), \operatorname{skew}\left(\nabla u_{, t}-P_{, t}\right)\right\rangle+\left\langle\widetilde{\mathbb{C}}_{\text {micro }} \operatorname{sym} P_{, t}, \operatorname{sym} P_{, t}\right\rangle \\
& +\mu \frac{L_{c}^{2}}{2}\left\langle\widetilde{\mathbb{U}}_{\text {aniso }} \operatorname{Curl} P_{, t}, \operatorname{Curl} P_{, t}\right\rangle
\end{aligned}
$$


and to the same potential energy density as in the previous subsection. A justification of a such choice is that in the limit case $P=\nabla u$, the variational formulation should be related to the equations of the linear theory of nonlocal elasticity introduced by Eringen [16] to fit the acoustical branch of elastic waves within the Brillouin zone in periodic one dimensional lattices [8]. We expect that the generalized model given in this section will improve the fitting of the dispersion curves, at least up to a value of the wave number compatible with the size of the microstructure of a considered microstructured material.

The equations of this model are

$$
\begin{gathered}
\rho u_{, t t}-\operatorname{Div}\left[\widetilde{\mathbb{C}}_{e} \cdot \operatorname{sym}\left(\nabla u, t t-P_{, t t}\right)+\widetilde{\mathbb{C}}_{c} \cdot \operatorname{skew}\left(\nabla u, t t-P_{, t t}\right)\right]= \\
\operatorname{Div}\left[\mathbb{C}_{e} \cdot \operatorname{sym}(\nabla u-P)+\mathbb{C}_{c} \cdot \operatorname{skew}(\nabla u-P)\right]+f \\
\mu L_{c}^{2} \operatorname{Curl}\left[\widetilde{\mathbb{L}}_{\text {aniso }} \cdot \operatorname{Curl} P_{, t t}\right]-\widetilde{\mathbb{C}}_{e} \cdot \operatorname{sym}\left(\nabla u_{, t t}-P_{, t t}\right)-\widetilde{\mathbb{C}}_{c} \cdot \operatorname{skew}\left(\nabla u_{, t t}-P_{, t t}\right)+\widetilde{\mathbb{C}}_{\text {micro }} \cdot \operatorname{sym} P_{, t t}= \\
-\mu L_{c}^{2} \operatorname{Curl}\left[\mathbb{L}_{\text {aniso }} \cdot \operatorname{Curl} P\right]+\mathbb{C}_{e} \cdot \operatorname{sym}(\nabla u-P)+\mathbb{C}_{c} \cdot \operatorname{skew}(\nabla u-P)-\mathbb{C}_{\text {micro }} \cdot \operatorname{sym} P+M .
\end{gathered}
$$

We assume that the remaining constants of the model satisfy the conditions from the above section.

In these hypotheses, the corresponding bilinear form defined by the right-hand side of the system of partial differential equations, i.e. $\mathcal{W}_{1}:\left(H_{0}^{1}(\Omega) \times H_{0}(\operatorname{Curl} ; \Omega)\right) \times\left(H_{0}^{1}(\Omega) \times H_{0}(\operatorname{Curl} ; \Omega)\right) \rightarrow \mathbb{R}$

$$
\begin{gathered}
\mathcal{W}_{1}((u, P),(\varphi, \Phi))=\int_{\Omega}\left(\rho\langle u, \varphi\rangle+\left\langle\widetilde{\mathbb{C}}_{\mathrm{e}} \cdot \operatorname{sym}(\nabla u-P), \operatorname{sym}(\nabla \varphi-\Phi)\right\rangle+\left\langle\widetilde{\mathbb{C}}_{\mathrm{c}} \cdot \operatorname{skew}(\nabla u-P), \operatorname{skew}(\nabla \varphi-\Phi)\right\rangle\right. \\
\left.+\left\langle\widetilde{\mathbb{C}}_{\text {micro }} \cdot \operatorname{sym} P, \operatorname{sym} \Phi\right\rangle+\mu L_{c}^{2}\left\langle\widetilde{\mathbb{L}}_{\text {aniso }} \text {. Curl } P, \operatorname{Curl} \Phi\right\rangle\right) d v
\end{gathered}
$$

remains bounded. Regarding its coercivity we point out that we have to impose, beside the conditions imposed in the last section, that $\widetilde{\mathbb{C}}_{\text {micro }}$ is positive definite.

Under this additional assumption, using the properties of the other constitutive tensors, we obtain that there is a $c>0$ such that

$$
\mathcal{W}_{1}(w, w) \geq c \int_{\Omega}\left(\|u\|^{2}+\|\operatorname{sym}(\nabla u-P)\|^{2}+\|\operatorname{sym} P\|^{2}+\|\operatorname{Curl} P\|^{2}\right) d v
$$

for all $w=(u, P) \in H_{0}^{1}(\Omega) \times H_{0}(\operatorname{Curl} ; \Omega)$, which means that there is a $c>0$ such that

$$
\mathcal{W}_{1}(w, w) \geq c \int_{\Omega}\left(\|\operatorname{sym} \nabla u\|^{2}+\|\operatorname{sym} P\|^{2}+\|\operatorname{Curl} P\|^{2}\right) d v
$$

for all $w=(u, P) \in H_{0}^{1}(\Omega) \times H_{0}(\operatorname{Curl} ; \Omega)$.

Let us recall the following result [51, 49, 50, 6]:

Theorem 3.1. There exists a positive constant $C$, only depending on $\Omega$, such that for all $P \in \mathrm{H}_{0}(\mathrm{Curl} ; \Omega)$ the following estimates hold:

$$
\|P\|_{H(\mathrm{Curl})}^{2}:=\|P\|_{L^{2}(\Omega)}^{2}+\|\operatorname{Curl} P\|_{L^{2}(\Omega)}^{2} \leq C\left(\|\operatorname{sym} P\|_{L^{2}(\Omega)}^{2}+\|\operatorname{Curl} P\|_{L^{2}(\Omega)}^{2}\right) .
$$

While in the model introduced in the previous section, we have used only the Poincaré inequality and Korn inequality to show the coercivity of the bilinear form from the left-hand side of the variational form of the boundary initial-value problem, in the model proposed in this section the estimate in Theorem 3.1 is essential. Indeed, using also the Korn inequality, we obtain that $\mathcal{W}_{1}$ is coercive for this model, too. Therefore, under the additional assumption that $\widetilde{\mathbb{C}}_{\text {micro }}$ is positive definite, a similar analysis as in the previous section shows that the model presented in this section is well-posed and the solution $(u, P)$ belongs to $C^{2}\left([0, T] ; H_{0}^{1}(\Omega) \times H_{0}(\mathrm{Curl} ; \Omega)\right)$.

The positive definiteness of $\mathbb{C}_{e}, \mathbb{C}_{c}, \mathbb{C}_{\text {micro }}, \mathbb{L}_{\text {aniso }}$ or $\widetilde{\mathbb{C}}_{\mathrm{c}}$ is still not necessary in order to have a well-posed model.

\section{Final remarks}

An approach based on the semigroup of linear operators may not lead to an existence result, even of a weak solution, without an a priori assumption on the compatibility of the domains of the operators defined by 
the left and right-hand side, respectively, of the system of partial differential equations (which are Banach spaces endowed with the corresponding graph-norms, since the operators generate $C_{0}$-contractive semigroups in $L^{2}(\Omega) \times L^{2}(\Omega)$ ). We expect to have the same difficulties when the Galerkin method is used. However, for a model considering that the elastic tensors $\mathbb{C}_{e}, \mathbb{C}_{c}, \mathbb{C}_{\text {micro }}$ and $\mathbb{L}_{\text {aniso }}$ vanish, the existence follows using techniques introduced in 53, 54]. In fact, in this particular case, the existence is based on the fact that the operator acting on $\left(u_{, t t}, P_{, t t}\right)$ is invertible, since it is of the form $\mathrm{Id}-\mathcal{A}$, where $\mathcal{A}$ generates a $C_{0}$-contractive semigroup in an appropiate Hilbert space, see [24].

Further, assuming that $\widetilde{\mathbb{C}}_{\text {micro }}$ is positive definite, the model remains well-posed also in the quasistatic case, i.e the situation when $\left\|P_{, t}\right\|$ and $\left\|u_{, t}\right\|$ are not present in the expression of the kinetic energy.

Another remark is that the first model considered in this paper remains well-posed also when the characteristic length scale $L_{c}$ tends to zero, i.e. Curl $P_{, t}$ and $\operatorname{Curl} P$ are not present in the kinetic energy and in the potential energy density, respectively. The solution will belong to $C^{2}\left([0, T] ; H_{0}^{1}(\Omega) \times L^{2}(\Omega)\right)$ as long as the forces are in $C\left([0, T] ; H^{-1}(\Omega) \times L^{2}(\Omega)\right)$ and the initial conditions are assumed to be in $H_{0}^{1}(\Omega) \times L^{2}(\Omega)$ (see also [28] for a related model). Note also that for $L_{c} \rightarrow 0$ we do not prescribe $P$ on the boundary.

We are not able to say the same for the second model, in the case $L_{c} \rightarrow 0$, since the bilinear form $\mathcal{W}_{1}$ may not be coercive, in general. However, when $\mathbb{C}_{\mathrm{c}}=0$ and $\widetilde{\mathbb{C}}_{\mathrm{c}}=0, L_{c} \rightarrow 0$ and $\left\|P_{, t}\right\|$ is not taken into account in the form of the kinetic energy, the problem involves actually only the functions $u$ and $\operatorname{sym} P$. Therefore, in this situation and when $\widetilde{\mathbb{C}}_{\text {micro }}$ is positive definite, if $\operatorname{sym} P(0)$, sym $P, t(0) \in L^{2}(\Omega), u(0), u_{, t}(0) \in H_{0}^{1}(\Omega), u=0$ on the boundary, $P$ is also prescribed on the boundary, and the forces are in $C\left([0, T] ; H^{-1}(\Omega) \times L^{2}(\Omega)\right)$, there exists a unique solution $(u, \operatorname{sym} P) \in C^{2}\left([0, T] ; H_{0}^{1}(\Omega) \times L^{2}(\Omega)\right)$ of the problem in $u$ and sym $P$. We do not have information about skew $P$, since it is not involved in the equations and clearly it may not be uniquely determined, in order to satisfy also the initial and the boundary conditions for the full $P$.

\section{Acknowledgement}

We are sincerely grateful to the reviewers for their insightful comments which helped improve the paper. The work of I.D. Ghiba was supported by Alexandru Ioan Cuza University of Iasi (UAIC) under the grant GI-17458, within the internal grant competition for young researchers.

\section{References}

[1] A. Aivaliotis, A. Daouadji, G. Barbagallo, D. Tallarico, P. Neff, and A. Madeo. Low-and high-frequency Stoneley waves, reflection and transmission at a Cauchy/relaxed micromorphic interface, arXiv preprint arXiv:1810.12578 2018

[2] R.A. Adams. Sobolev Spaces., volume 65 of Pure and Applied Mathematics. Academic Press, London, 1. edition, 1975.

[3] H. Askes, D. Nguyen, and A. Tyas. Increasing the critical time step: micro-inertia, inertia penalties and mass scaling. Comp. Mech., 47(6):657-667, 2011.

[4] G. Barbagallo, A. Madeo, M.V. d'Agostino, R. Abreu, I.D. Ghiba, and P. Neff. Transparent anisotropy for the relaxed micromorphic model: macroscopic consistency conditions and long wave length asymptotics. Int. J. Solids Struct., 120:7-30, 2017.

[5] G. Barbagallo, D. Tallarico, M.V. D'Agostino, A. Aivaliotis, P. Neff, A. Madeo. Relaxed micromorphic model of transient wave propagation in anisotropic band-gap metastructures. Int. J. Solids Struct., https://doi.org/10.1016/j.ijsolstr.2018.11.033, 2018.

[6] S. Bauer, P. Neff, D. Pauly, and G. Starke. Dev-Div and DevSym-DevCurl inequalities for incompatible square tensor fields with mixed boundary conditions. ESAIM: COCV, 22(1):112-133, 2016.

[7] T. Bennett, I.M. Gitman, and H. Askes. Elasticity theories with higher-order gradients of inertia and stiffness for the modelling of wave dispersion in laminates. Int. J. Fract., 148(2):185-193, 2007.

[8] L. Brillouin. Wave propagation in periodic structures: electric filters and crystal lattices. Courier Corporation, 2003.

[9] K. Chełmiński and S. Owczarek. Renormalized solutions in thermo-visco-plasticity for a Norton-Hoff type model. Part I: The truncated case. Nonlinear Anal. Real World Appl., 28:140-152, 2016.

[10] W. Chen and J. Fish. A dispersive model for wave propagation in periodic heterogeneous media based on homogenization with multiple spatial and temporal scales. J. Applied Mech., 68(2):153-161, 2001.

[11] W.D. Claus and A.C. Eringen. Three dislocation concepts and micromorphic mechanics. In Developments in Mechanics, Proceedings of the 12th Midwestern Mechanics Conference, volume 6, pages 349-358. Midwestern, 1969. 
[12] E. Cosserat and F. Cosserat. Théorie des corps déformables. Librairie Scientifique A. Hermann et Fils (engl. translation by D. Delphenich 2007, pdf available at http://www.mathematik.tudarmstadt.de/fbereiche/analysis/pde/staff/neff/patrizio/Cosserat.html), reprint 2009 by Hermann Librairie Scientifique, ISBN 978270566920 1, Paris, 1909.

[13] M.V. d'Agostino, G. Barbagallo, I.D. Ghiba, B. Eidel, P. Neff, and A. Madeo. Effective description of anisotropic wave dispersion in mechanical meta-materials via the relaxed micromorphic model. arXiv preprint arXiv:1709.07054, 2018.

[14] M.V. d'Agostino, G. Barbagallo, I.D. Ghiba, A. Madeo, and P. Neff. A panorama of dispersion curves for the weighted isotropic relaxed micromorphic model. Z. Angew. Math. Mech., 97(11):1436-1481, 2017.

[15] D. De Domenico and H. Askes. A new multi-scale dispersive gradient elasticity model with micro-inertia: Formulation and-finite element implementation. Int. J. Numer. Meth. in Eng., 108(5):485-512, 2016.

[16] A.C. Eringen. Linear theory of nonlocal elasticity and dispersion of plane waves. Int. J. Eng. Sci., 10(5):425-435, 1972.

[17] A.C. Eringen. Microcontinuum Field Theories. Springer, Heidelberg, 1999.

[18] A.C. Eringen and D.G.B. Edelen. On nonlocal elasticity. Int. J. Eng. Sci., 10(3):233-248, 1972.

[19] A.C. Eringen and E.S. Suhubi. Nonlinear theory of simple micro-elastic solids. Int. J. Eng. Sci., 2:189-203, 1964.

[20] A. Blanco, E. Chomski, S.Grabtchaket, M. Ibisate, S. John et al., Large-scale synthesis of a silicon photonic crystal with a complete three-dimensional bandgap near 1.5 micrometres. Nature, 405(6785):437-440, 2000.

[21] Z. Liu, Z. Xixiang, M. Yiwei, Y. Y. Zhu, Y. Zhiyu, C. T. Chan, and S. Ping, Locally resonant sonic materials. Science, 289(5485):1734-1736, 2000.

[22] S. Forest and R. Sievert. Nonlinear microstrain theories. Int. J. Solids Struct., 43:7224-7245, 2006.

[23] P. Germain. The method of virtual power in continuum mechanics. Part 2: Microstructure. SIAM J. Appl. Math., 25(3):556$575,1973$.

[24] I. D. Ghiba, P. Neff, A. Madeo, L. Placidi, and G. Rosi. The relaxed linear micromorphic continuum: Existence, uniqueness and continuous dependence in dynamics. Math. Mech. Solids, 20:1171-1197, 2015.

[25] V. Girault and P.A. Raviart. Finite Element Approximation of the Navier-Stokes Equations., volume 749 of Lect. Notes Math. Springer, Heidelberg, 1979.

[26] I.M. Gitman, H. Askes, and E.C. Aifantis. Gradient elasticity with internal length and internal inertia based on the homogenisation of a representative volume element. J. Mech. Behav. Biomed. Mater., 18(1):1-16, 2007.

[27] I. Hlaváček and M. Hlaváček. On the existence and uniqueness of solutions and some variational principles in linear theories of elasticity with couple-stresses. I: Cosserat continuum. II: Mindlin's elasticity with micro-structure and the first strain gradient. J. Apl. Mat., 14:387-426, 1969.

[28] M. Hlaváček. A continuum theory for isotropic two-phase elastic composites. Int. J. Solids Struct., 11:1137-1144, 1975.

[29] D. Ieşan. Extremum principle and existence results in micromorphic elasticity. Int. J. Eng. Sci., 39:2051-2070, 2001.

[30] D. Ieşan. On the micromorphic thermoelasticity. Int. J. Eng. Sci., 40:549-567, 2002.

[31] J. Jeong and P. Neff. Existence, uniqueness and stability in linear Cosserat elasticity for weakest curvature conditions. Math. Mech. Solids, 15(1):78-95, 2010.

[32] R. Leis. Initial Boundary Value problems in Mathematical Physics. Teubner, Stuttgart, 1986.

[33] A. Madeo, M. Collet, M. Miniaci, K. Billon, Morvan M. Ouisse, and P. Neff. Modeling phononic crystals via the weighted relaxed micromorphic model with free and gradient micro-inertia. J. Elasticity, 130(1):59-83, 2018.

[34] A. Madeo, P. Neff, E. Aifantis, G. Barbagallo, and M.V. d'Agostino. On the role of micro-inertia in enriched continuum mechanics. Proc. R. Soc. A, 473(2198):20160722, 2017.

[35] A. Madeo, P. Neff, G. Barbagallo, M.V. d'Agostino, and I.D. Ghiba. A review on wave propagation modeling in band-gap meta-materials via enriched continuum models. In Mathematical Modelling in Solid Mechanics, pages 89-105. Springer, 2017.

[36] A. Madeo, P. Neff, M.V. d'Agostino, and G. Barbagallo. Complete band gaps including non-local effects occur only in the relaxed micromorphic model. Comptes Rendus Mécanique, 344(11-12):784-796, 2016.

[37] A. Madeo, P. Neff, I. D. Ghiba, L. Placidi, and G. Rosi. Wave propagation in relaxed linear micromorphic continua: modelling meta-materials with frequency band-gaps. Cont. Mech. Therm., 27:551-570, 2015.

[38] A. Madeo, P. Neff, I.D. Ghiba, and G. Rosi. Reflection and transmission of elastic waves in non-local band-gap meta-materials: a comprehensive study via the relaxed micromorphic model. J. Mech. Physics of Solids, 95:441-479, 2016.

[39] R.D. Mindlin. Micro-structure in linear elasticity. Arch. Rat. Mech. Anal., 16:51-77, 1964.

[40] P. Neff, On Korn's first inequality with nonconstant coefficients., Proc. Roy. Soc. Edinb. A, 132:221-243, 2002

[41] P. Neff. Existence of minimizers for a finite-strain micromorphic elastic solid. Proc. Roy. Soc. Edinb. A, 136:997-1012, 2006.

[42] P. Neff, M. Bîrsan, and F. Osterbrink. Existence theorem for geometrically nonlinear cosserat micropolar model under uniform convexity requirements. J. Elasticity, 121(1):119-141, 2015.

[43] P. Neff and K. Chełmiński. Well-posedness of dynamic Cosserat plasticity. Appl. Math. Optim., 56:19-35, 2007.

[44] P. Neff and S. Forest. A geometrically exact micromorphic model for elastic metallic foams accounting for affine microstructure. Modelling, existence of minimizers, identification of moduli and computational results. J. Elasticity, 87:239-276, 2007. 
[45] P. Neff, I. D. Ghiba, M. Lazar, and A. Madeo. The relaxed linear micromorphic continuum: well-posedness of the static problem and relations to the gauge theory of dislocations. Q. J. Mech. Appl. Math., 68:53-84, 2015.

[46] P. Neff, I. D. Ghiba, A. Madeo, L. Placidi, and G. Rosi. A unifying perspective: the relaxed linear micromorphic continuum. Cont. Mech. Therm., 26:639-681, 2014.

[47] P. Neff, A. Madeo, G. Barbagallo, M.V. d'Agostino, R. Abreu, and I.D. Ghiba. Real wave propagation in the isotropic-relaxed micromorphic model. Proc. R. Soc. A, 473(2197):20160790, 2017.

[48] P. Neff and I. Münch. Curl bounds Grad on SO(3). ESAIM: Control, Optimisation and Calculus of Variations, 14(1):148-159, 2008.

[49] P. Neff, D. Pauly, and K.J. Witsch. A canonical extension of Korn's first inequality to H(Curl) motivated by gradient plasticity with plastic spin. C. R. Acad. Sci. Paris, Ser. I, 349:1251-1254, 2011.

[50] P. Neff, D. Pauly, and K.J. Witsch. Maxwell meets Korn: a new coercive inequality for tensor fields in $\mathbb{R}^{N \times N}$ with squareintegrable exterior derivative. Math. Methods Appl. Sci., 35:65-71, 2012.

[51] P. Neff, D. Pauly, and K.J. Witsch. Poincaré meets Korn via Maxwell: Extending Korn's first inequality to incompatible tensor fields. J. Differential Equations, 258:1267-1302, 2015.

[52] J.W. Nunziato and S.C. Cowin. A nonlinear theory of elastic materials with voids. Arch. Rat. Mech. Anal., 72:175-201, 1979.

[53] R. Picard. A structural observation for linear material laws in classical mathematical physics. Math. Meth. Appl. Sci., 32:1768-1803, 2009.

[54] R. Picard. Mother operators and their descendants. J. Math. Anal. Appl., 403: 54-62, 2013.

[55] R. Picard, S. Trostorff, and M. Waurick. A functional analytic perspective to delay differential equations. Operators and Matrices, 8 (1): 217-236, 2014.

[56] R. Picard, S. Trostorff, and M. Waurick. On some models for elastic solids with micro-structure. Z. Angew. Math. Mech., 95(7):664-689, 2014.

[57] M.B. Rubin, Ph. Rosenau, and O. Gottlieb. Continuum model of dispersion caused by an inherent material characteristic length. J. Appl. Phys., 77(8):4054-4063, 1995.

[58] E. Soós. Uniqueness theorems for homogeneous, isotropic, simple elastic and thermoelasticmaterials having a microstructure. Int. J. Eng. Sci., 7:257-268, 1969.

[59] C. Teodosiu. Discussion on Papers by A.C. Eringen and W.D. Claus, Jr., and N. Fox. In J.A. Simmons, R. de Wit, and R. Bullough, editors, Fundamental Aspects of Dislocation Theory., volume 1 of Nat. Bur. Stand. (U.S.), Spec. Publ., pages 1054-1059. Spec. Publ., 1970.

[60] Z.P. Wang and C.T. Sun. Modeling micro-inertia in heterogeneous materials under dynamic loading. Wave Motion, 36(4):473485,2002 